\title{
Preliminary Idea for a Converged Fixed and Mobile Network Infrastructure with 5G using Radio-over-Fiber Technology and an Opto-Electronic Oscillator in the Millimeter-Wave Range
}

\author{
Mehmet Alp Ilgaz, Bostjan Batagelj, Member, IEEE \\ University of Ljubljana, Faculty of Electrical Engineering, Radiation and Optics Laboratory, \\ Trzaska 25, 1000 Ljubljana, Slovenia \\ Tel: +38614768 400,e-mail: Mehmet.Ilgaz@fe.uni-lj.si
}

\begin{abstract}
Radio-over-Fiber (RoF) is becoming increasingly popular for the transportation of wireless signals due to it having a couple of advantages in the millimeter-wave communication architecture. This paper focuses on bringing a new approach to produce high-frequency signals in the millimeter-wave region for Radio-over-Fiber technology.
\end{abstract}

It also gives information regarding an Opto-Electronics Oscillator (OEO) that has been designed for $3 \mathrm{GHz}$ to produce signals with long-term stability, a high side-mode suppression ratio with a low phase noise and can be a key component for $5 \mathrm{G}$ converged networks.

Keywords: 5G Technology, single-loop opto-electronic oscillator, feedback control loop, long-term stability, side-mode suppression ratio, low phase noise

\section{INTRODUCTION}

Of all today's technology challenges [1] in optical communications, the Radio-over-Fiber technology is a very promising solution for transporting wireless signals and increasing the capacity of $5 \mathrm{G}$ mobile-access networks [2]. In the RoF system, radio- or even millimeter-wave signals can be transmitted via an optical fiber to a remote antenna and enable the convergence of fixed and mobile networks [3].

In the case of a practical network with a large number of remote stations and very few central stations, RoF transmission has plenty of benefits over current data-transfer techniques [4]. The use of an optical fiber technique is generally an advantage when transmitting spectrally broad signals.

The frequency range of the millimeter waves can be defined between $30 \mathrm{GHz}$ and $300 \mathrm{GHz}$, which can be used for short-range communications in $5 \mathrm{G}$.

\section{OEO IN ROF APPLICATIONS}

The opto-electronics oscillator is a well-known technology to generate spectrally pure signals up to $75 \mathrm{GHz}$ [5]. This is a new technique for high-frequency signal generation in the millimeter wave range that was invented by Yao and Maleki in the mid-1990s [5]. Due to it having the low phase-noise characteristic of an OEO (the reason for this is to have a high Q factor), it is believed that the OEO can bring positive advantages to RoF applications.

The OEO can be used in many applications where a high-performance oscillator is required, such as satellite communications links [6] or communication wireless links [7]. The most significant advantage of the OEO is that the phase noise does not depend on the frequency [6]. In other oscillators, such as quartz oscillators or dielectric resonators, the phase noise increases if we go to higher frequencies [6].

In the literature other ways to generate microwave or millimeter wave signals, such as optical heterodyning [8] or a dual-loop OEO with an electro-absorption modulator [9], have been described. With the latter method with the helping of controlling the gain for each loop there is a $20-\mathrm{dB}$ less noise performance than with a single-loop $\mathrm{OEO}$ at low frequencies $(<1 \mathrm{kHz})$. Using an OEO incorporating a carrier suppression noise-reduction technique is another way to generate microwave signals [10]. With this technique, the noise performance of the OEO was improved by about $20 \mathrm{~dB}[10]$.

Figure 1 shows how the OEO can be implemented in a RoF system in order to simplify the network's basestations [3]. This single-loop OEO can be improved by using a feedback control loop in order to have a better stability and a low phase noise [11]. The oscillator signal is transmitted from the central-station to base-station 
with separate optical fiber. At the base-station, the optical signal is converted to electrical domain on the photodiode, creating a RF signal suitable for frequency conversion of the data signal in the mixers.

The technology which uses separate optical lines for the data signal and for mixing signal is displayed in Figure 1. All optical lines can also be joined in a single optical fiber using wavelength-division multiplexing (WDM) technology.

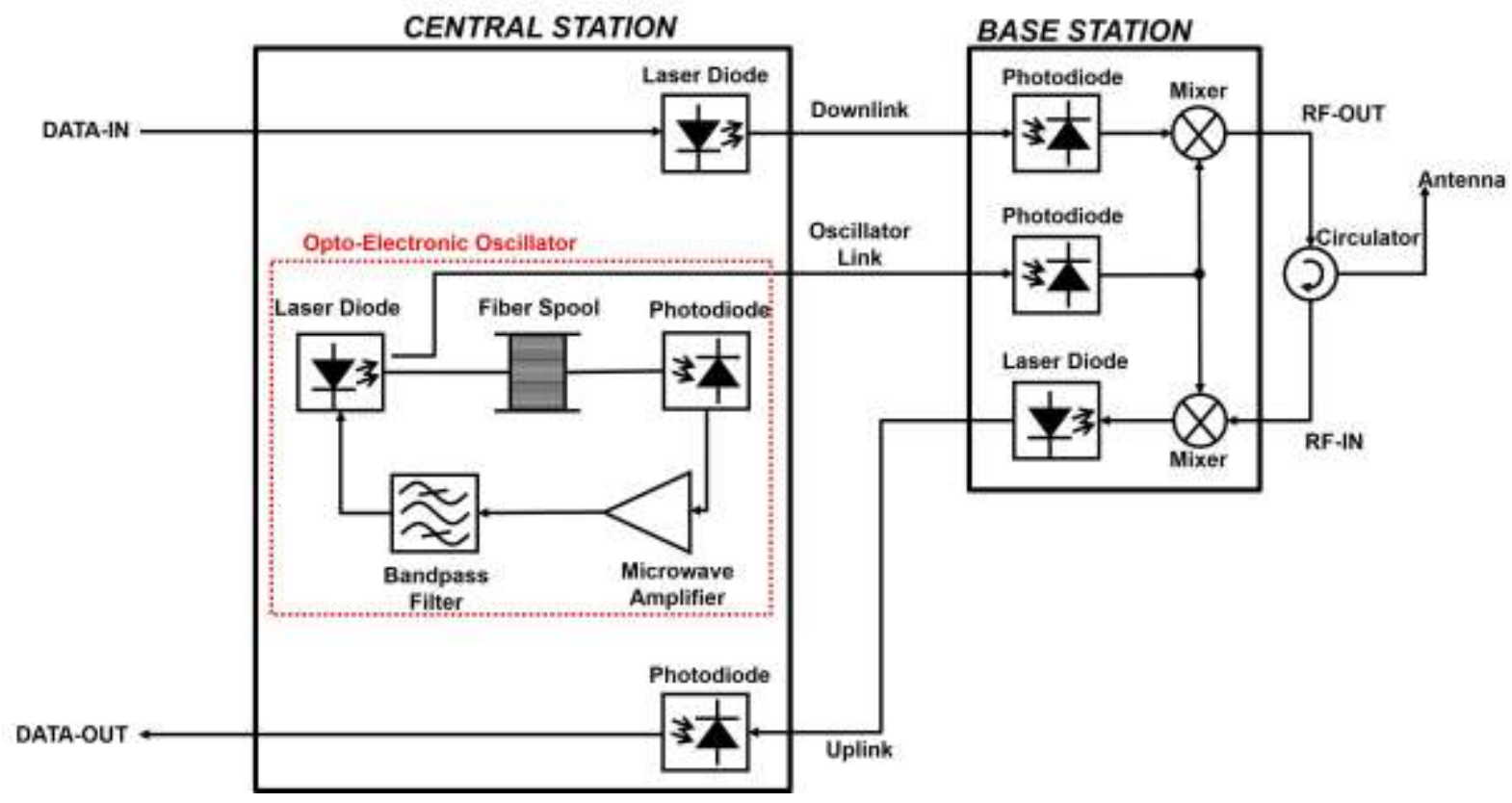

Figure 1. Idea of a Radio-over-Fiber System with an Opto-electronic Oscillator.

\section{SINGLE-LOOP OEO}

This paper is composed of studies on a single-loop OEO used to produce signals at a frequency of $3 \mathrm{GHz}$. This single-loop OEO consists of electronic and optical parts. The OEO that was proposed for the previous studies is composed of an amplifier and a resonator as a usual electrical oscillator. Figure 2 shows the schematic of the OEO with a feedback control loop [11].

There are two main issues that affect the performance of the OEO. These are the long-term stability and the multi-mode operation of the OEO [6]. The refractive index of the optical fiber is a temperature-dependent parameter [12]. This means that when the temperature changes the refractive index changes too, which causes instability of the frequency. There have been some solutions to overcome this problem, such as stabilization of the optical fiber and a bandpass filter [12]. There was a method described in [11] using a feedback control loop to stabilize the frequency. In this method, a temperature coefficient of $0.2 \mathrm{ppm} / \mathrm{K}$ was achieved. Figure 2 shows the OEO with a feedback control loop to allow long-term stabilization.

The feedback control loop with additional phase modulation has an almost 160 -times-smaller $(0.05 \mathrm{ppm} / \mathrm{K})$ frequency drift than the single-loop OEO without a feedback control loop [13].

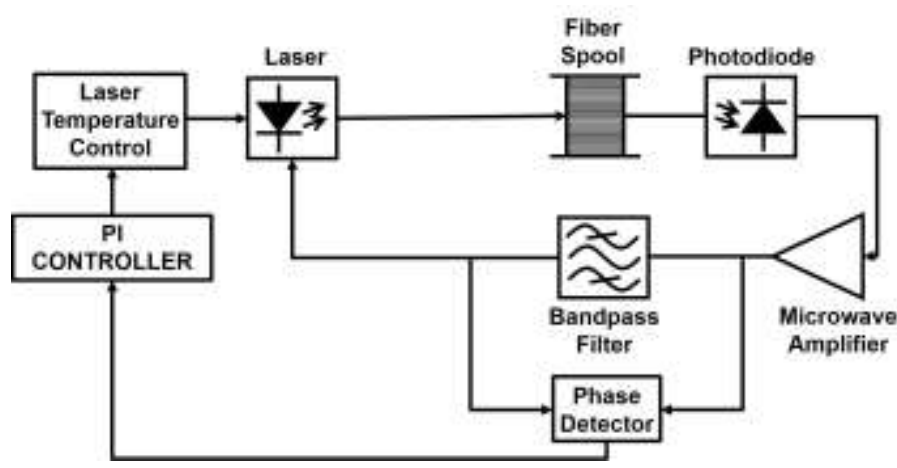


Figure 2. Schematic of the OEO with a feedback control loop that was established in the lab. The control loop has a Proportional Integral Controller that is used to drive the temperature of the laser.

In addition, the phase noise is related to the length of the optical path, with the phase noise being inversely proportional to the optical length [6]. Figure 3 shows the phase noise of two different optical paths. According to the results, it is clear that when we increase the length of the optical path, the phase noise decreases smoothly.

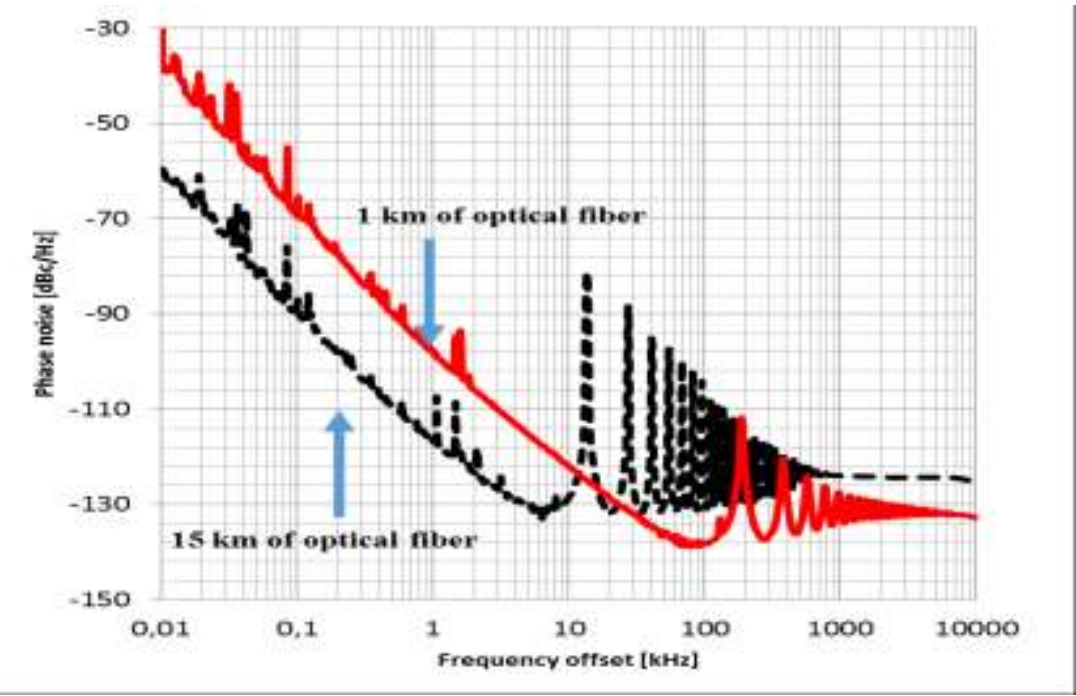

Figure 3. Phase-noise result for a single-loop OEO with two different optical lengths (1 km and $15 \mathrm{~km})$ [6].

The second issue with the OEO is its multi-mode operation [5]. This means that the OEO produces signals with a comb frequency spectrum. In order to decrease the multi-modes of the oscillator, a microwave bandpass filter is used. With this filter, there is only one frequency value selected, while the others are attenuated and can still be detectable. In the literature, some methods have been invented in order to overcome these unwanted modes. A dual-loop OEO [9] or a multi-loop OEO [14] can be used to suppress these spurious frequency components. But this review paper focuses on the methods to improve the SMSR by using a single-loop OEO due to it having a simple and basic solution.

Figure 4 shows the power and frequency (ppm) of the multi-modes of the OEO when a cavity filter is used at 3 GHz. However, side modes cannot be destroyed by cavity filter, but they are evidently attenuated. These experiments in Figure 3 and Figure 4 were conducted in our laboratory.

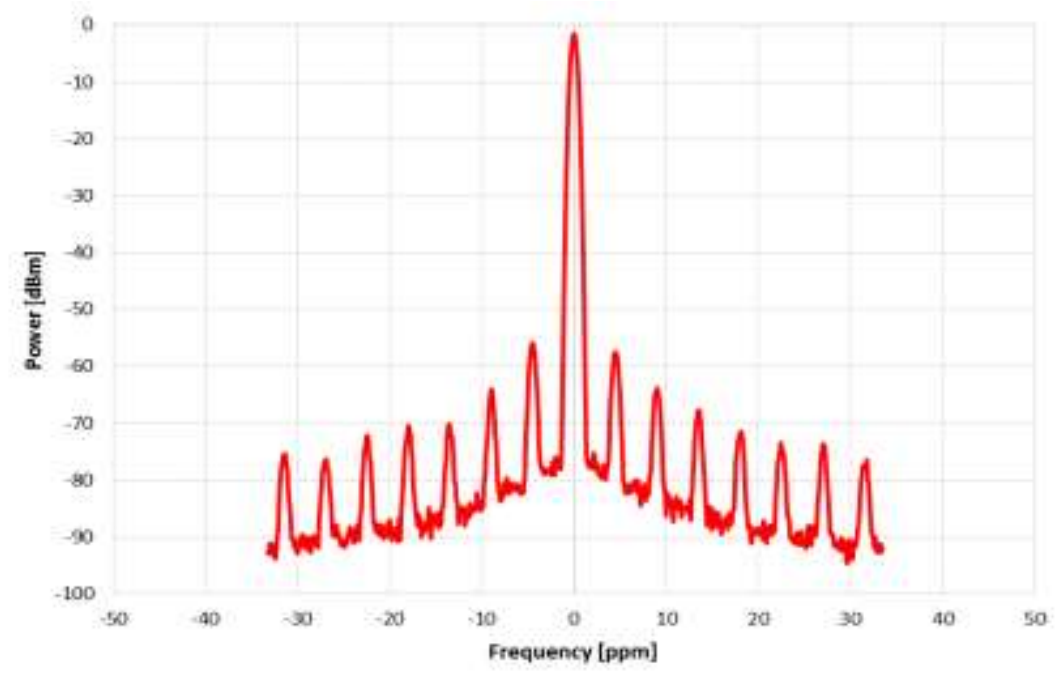

Figure 4. Frequency spectrum of the OEO with the cavity filter at $3 \mathrm{GHz}$. 
Moreover, there is another implementation on the OEO in order to reduce the power of the side modes. With this approach there is a Quality Multiplier (QM) implemented on the feedback of the OEO loop [15]. In this method, a microwave electrical circuit is placed to a bandpass filter in the feedback loop. The aim of this technique is to reduce the bandwidth of the OEO loop by using a quality multiplier to a bandpass filter in order to improve the SMSR. The feedback loop consists of a Bandpass Filter, an Adjustable Gain and an Adjustable Phase Shift. In [15] there is an experimental setup to show the how the QM can affect the SMSR.

According to the results, there is a significant improvement in the SMSR of $20 \mathrm{~dB}$. Unfortunately, with the QM the phase noise is also increased, which is an undesired effect on the OEO. The phase noise increases by $4 \mathrm{~dB}$ with a 1-kHz offset. The most significant advantage of implementing the QM is that the components of the QM can be easily found and implemented on the OEO. On the other hand, optical filters with a high Q factor cannot be obtained easily [15].

\section{CONCLUSION}

In this paper a new method was described for the generation of high-frequency signals in the millimeter-wave range. As described before it was reported that the OEO is a new type of oscillator that can be used to produce spectrally pure signals up to $75 \mathrm{GHz}$. In the single-loop OEO, the feedback control loop allows a lower frequency drift and a higher stability compared to other oscillators.

The SMSR is another criteria that should be taken into consideration. There are two methods that are described to decrease the effect of side modes. The first method is placing an ECPS into the feedback control loop [13]. With this method there is only a 5-dB improvement in the SMSR with no observable increase in the phase noise. On the other hand, with the second method there is a QM implemented in the feedback loop, which allows a 20$\mathrm{dB}$ improvement on the SMSR with an unwanted increase in the phase noise [15].

\section{ACKNOWLEDGEMENTS}

The authors would like to express their gratitude to the company InLambda BDT d.o.o. for the research equipment and devices. This work is part of the FiWIN5G Innovative Training Network, which has received funding from the European Union's Horizon 2020 Research and Innovation Programme 2014-2018 under grant agreement No.642355.

\section{REFERENCES}

[1] B.Batagelj, et al.: Research challenges in optical communications towards 2020 and beyond, J. Informacije MIDEM, vol. 44, no. 3, pp. 177-184, Sept. 2014.

[2] Nathan J. Gomes, et al.: Radio-over-fiber transport for the support of wireless broadband services, $J$. Optical Netw., vol.8, no. 2, pp. 156-178, Feb. 2009.

[3] Bostjan Batagelj, et al.: Convergence of fixed and mobile networks by radio over fibre technology, $J$. Informacije MIDEM, vol. 41, no. 2, pp. 144-149, June 2011.

[4] John E. Mitchell: Radio-over-Fiber (RoF) Networks, in Broadband Access Networks: Technologies and Deployments, A.Shami, M.Maier, C. Assi, Ed., Springer, 2009.

[5] X. S. Yao, L. Maleki: Optoelectronic microwave oscillator, J. Optical Soc. Am. B, vol. 13, no. 8, pp. $1725-$ 1735, Aug. 1996.

[6] B. Batagelj, L. Bogataj and M. Vidmar: Key properties and design issues or an opto-electronic oscillator, in Proc. ICTON 2015, Budapest, Hungary, July 2015, paper Mo.C5.4.

[7] FIWIN5G: Fiber-Wireless Integrated Networks for $5^{\text {th }}$ Generation delivery, http://www.fiwin5g.eu

[8] J. Yao: Microwave photonics, J. Lightwave Technol., vol. 27, no. 3, pp. 314-335, Feb. 2009.

[9] M. Shin, P.S.Devgan, V.S.Grigoryan, and Prem Kumar: Low Phase-Noise 40GHz Optical Pulses from a Self-Starting Electroabsorption-Modulator-Based Optoelectronic Oscillator, in Proc. OFC 2006, Anaheim, USA, Mar. 2006.

[10] X. S. Yao, L.Maleki, J.Dick: Opto-electronic oscillator incorporating carrier suppression noise reduction technique, in Proc.1999 Joint Meeting ETFT, Besancon, France, Apr.1999.

[11] L. Bogataj, et al: A feedback control loop for frequency stabilization in an opto-electronic oscillator, $J$. Lightwave Technol., vol. 32, no. 20, pp. 3690-3694, Oct. 2014.

[12] D. Eliyahu, K.Sariri, A.Kamran and M.Tokhmakhian: Improving short and long term frequency stability of the opto-electronic oscillator, in Proc. IEEE Int. Freq. Control Symp. And PDA Exhib, New Orleans, USA, May 2002. 
[13] L. Bogataj, et al: Improving the side-mode suppression ratio and reducing frequency drift in an optoelectronic oscillator with a feedback control loop and additional phase modulation, J. Lightwave Technol., vol. 34, no. 3. pp. 885-890, Feb. 2016.

[14] E. Shumakher and G. Eisenstein: A novel multiloop optoelectronic oscillator, IEEE Photonics Technol. Lett., vol. 20, no. 22, pp. 1881-1883, Nov. 2008.

[15] L. Bogataj, et al: Opto-electronic oscillator with quality multiplier, IEEE Trans. Microw. Theory and Techn., vol. 64, no. 2, pp. 663-668, Feb. 2016. 